\title{
FANO HYPERSURFACES WITH ARBITRARILY LARGE DEGREES OF IRRATIONALITY
}

\author{
NATHAN CHEN ${ }^{1}$ and DAVID STAPLETON ${ }^{2}$ \\ ${ }^{1}$ Department of Mathematics, Stony Brook University, Stony Brook, NY 11794, USA; \\ email: nathan.chen@ stonybrook.edu \\ 2 Department of Mathematics, UC San Diego, La Jolla, CA 92093, USA; \\ email: dstapleton@ucsd.edu
}

Received 29 January 2020; accepted 31 March 2020

\begin{abstract}
We show that complex Fano hypersurfaces can have arbitrarily large degrees of irrationality. More precisely, if we fix a Fano index $e$, then the degree of irrationality of a very general complex Fano hypersurface of index $e$ and dimension $\mathrm{n}$ is bounded from below by a constant times $\sqrt{n}$. To our knowledge, this gives the first examples of rationally connected varieties with degrees of irrationality greater than 3 . The proof follows a degeneration to characteristic $p$ argument, which Kollár used to prove nonrationality of Fano hypersurfaces. Along the way, we show that in a family of varieties, the invariant 'the minimal degree of a dominant rational map to a ruled variety' can only drop on special fibers. As a consequence, we show that for certain low-dimensional families of varieties, the degree of irrationality also behaves well under specialization.
\end{abstract}

2010 Mathematics Subject Classification: 14E05 (primary); 14J45 (secondary)

There has been a great deal of interest in studying questions of rationality of various flavors. Recall that an $n$-dimensional variety $X$ is rational if it is birational to $\mathbb{P}^{n}$ and ruled if it is birational to $\mathbb{P}^{1} \times Z$ for some variety $Z$. Let $X \subset \mathbb{P}_{\mathbb{C}}^{n+1}$ be a complex degree $d$ hypersurface. In [9], Kollár proved that when $X$ is very general and $d \geqslant(2 / 3)(n+3)$, then $X$ is not ruled (and thus not rational). Recently, these results were generalized and improved by Totaro [16] and subsequently by Schreieder [15]. Schreieder showed that when $X$ is very general and $d \geqslant \log _{2}(n)$ +2 , then $X$ is not even stably rational. In a positive direction, Beheshti and Riedl [2] proved that when $X$ is smooth and $n \geqslant 2^{d !}$, then $X$ is at least unirational, that is, dominated by a rational variety.

(c) The Author(s) 2020. This is an Open Access article, distributed under the terms of the Creative Commons Attribution licence (http://creativecommons.org/licenses/by/4.0/), which permits unrestricted re-use, distribution, and reproduction in any medium, provided the original work is properly cited. 
Given a variety $X$ whose nonrationality is known, one can ask if there is a way to measure 'how irrational' $X$ is. One natural invariant in this direction is the degree of irrationality, defined as

$$
\operatorname{irr}(X):=\min \left\{\delta>0 \mid \exists \text { a degree } \delta \text { dominant rational map } X \rightarrow \mathbb{P}^{n}\right\} .
$$

For instance, Bastianelli et al. [1] computed the degree of irrationality for very general hypersurfaces of degree $d \geqslant 2 n+1$ by using the positivity of the canonical bundle. Naturally, one is tempted to ask what can be proved about hypersurfaces with a negative canonical bundle.

Our main result gives the first examples of Fano varieties with arbitrarily large degrees of irrationality.

THEOREM A. Let $X_{n, d} \subset \mathbb{P}_{\mathbb{C}}^{n+1}$ be a very general hypersurface of dimension $n$ and degree $d$. If $d \geqslant n+1-\sqrt{n+2} / 4$, then

$$
\operatorname{irr}\left(X_{n, d}\right) \geqslant \frac{\sqrt{n+2}}{4} .
$$

In fact, we prove the stronger statement that the minimal degree map from $X_{n, e}$ to a ruled variety is bounded from below by $\sqrt{n+2} / 4$. These give the first examples of rationally connected varieties $X$ with $\operatorname{irr}(X) \geqslant 4$. (Irrational Fano varieties $X$ have $\operatorname{irr}(X) \geqslant 2$, and rational covers of degree 2 always admit birational involutions. Iskovskih and Manin's work [6] on the Noether-Fano method implies that a general smooth quartic threefold $X$ has a trivial birational automorphism group. Thus $\operatorname{irr}(X)=3$.)

The proof of Theorem A proceeds by extending ideas from Kollár's paper [9], using a specialization to characteristic $p>0$. This involves two main additions to the arguments in [9]. First, we use positivity considerations involving separation of points to show that the hypersurfaces constructed by Kollár do not admit lowdegree maps to ruled varieties. Our main technical result then asserts that such mappings, if they exist, behave well in families. Specifically, given a family of projective varieties over the spectrum of a discrete valuation ring (DVR), we prove that the minimal degree of a rational map to a ruled variety can only drop upon specialization. To be more precise, let $T$ be the spectrum of a DVR with generic point $\eta$ and residue field $\kappa$, and let $\mathscr{X}_{T}$ be an integral normal scheme that is flat and projective over $T$ such that $\mathscr{X}_{\eta}$ is geometrically integral. With this set-up in mind, we have the following theorem.

THEOREM B. If $\mathscr{X}_{\eta}$ admits a dominant and generically finite rational map to a ruled variety with degree $\leqslant d$, then so does every component $\mathscr{X}_{\kappa}^{\prime} \subset \mathscr{X}_{\kappa}^{\text {red }}$. 
A similar result holds when comparing the geometric generic fiber to the special fiber (see Theorem 1.1).

We give other applications of Theorem B concerning the behavior of the degree of irrationality in certain families. The last few years have seen major progress in understanding the behavior of rationality and stable rationality in families. Hassett et al. [5] showed that there are families of varieties where there is a dense set of rational fibers, but the very general member is irrational. Nicaise and Shinder [14] (respectively, Kontsevich and Tschinkel [12]) established that stable rationality (respectively, rationality) specializes in smooth projective families. The behavior of unirationality and the degree of irrationality in families is understood to a lesser extent. Applying Theorem B, we show that in certain families the degree of irrationality can only drop upon specialization.

Proposition C. Let $\pi: \mathscr{X} \rightarrow T$ be a smooth projective family of complex varieties over a smooth irreducible curve $T$ with marked point $0 \in T$. Assume that a very general fiber $\mathscr{X}_{t}$ is either:

(1) a surface with $H^{1,0}\left(\mathscr{X}_{t}\right)=0$; or

(2) a simply connected threefold with $\omega_{\mathscr{X}_{t}} \cong \mathcal{O}_{\mathscr{X}_{t}}$ (that is a strict Calabi-Yau threefold).

If $\operatorname{irr}\left(\mathscr{X}_{t}\right) \leqslant d$, then $\operatorname{irr}\left(\mathscr{X}_{0}\right) \leqslant d$.

By work of the first author [4], it is known that a very general abelian surface $A$ has $\operatorname{irr}(A) \leqslant 4$. From Proposition $\mathrm{C}$, we are able to deduce:

COROLlary D. Every complex abelian surface A has $\operatorname{irr}(A) \leqslant 4$.

In Section 1, we prove a generalized version of Theorem B, as well as Proposition C and Corollary D. In Section 2, we prove Theorem A. Throughout the paper, by variety we mean an integral separated scheme of finite type over a field (not necessarily algebraically closed). By very general, we mean away from the union of countably many proper closed subsets. If $\mathscr{X}$ is a scheme over $T$ and $k$ is a field with a map $\operatorname{Spec}(k) \rightarrow T$, by abuse of notation we write

$$
\mathscr{X}_{k}:=\mathscr{X} \times_{T} \operatorname{Spec}(k) \text {. }
$$

\section{Maps to ruled varieties specialize}

The goal of this section is to prove a slightly more general version of Theorem B. Let $T=\operatorname{Spec}(A)$ be the spectrum of a DVR with uniformizer $t$, fraction 
field $\eta=\operatorname{Frac}(A)$, and residue field $\kappa=A / t$. Let $\bar{\eta}$ and $\bar{\kappa}$ be their respective algebraic closures. Assume that $\mathscr{X}_{T} \rightarrow T$ is a flat projective morphism, and $\mathscr{X}_{T}$ is a normal integral scheme such that $\mathscr{X}_{\eta}$ is geometrically integral.

THEOREM 1.1. With the set-up above:

(1) If $\mathscr{X}_{\eta}$ admits a dominant generically finite rational map to a ruled variety

$$
\varphi: \mathscr{X}_{\eta} \rightarrow Z_{\eta} \times \mathbb{P}_{\eta}^{1}
$$

with $\operatorname{deg}(\varphi) \leqslant d$, then so does every component $\mathscr{X}_{\kappa}^{\prime} \subset \mathscr{X}_{\kappa}^{\mathrm{red}}$ (where $\mathscr{X}_{\kappa}^{\mathrm{red}}$ denotes the reduction of the special fiber).

(2) Suppose that $\mathscr{X}_{\kappa}^{\prime} \subset \mathscr{X}_{\kappa}$ is a component that is geometrically reduced and geometrically irreducible. If $\mathscr{X}_{\bar{\eta}}$ admits a dominant rational map to a ruled variety

$$
\varphi: \mathscr{X}_{\bar{\eta}} \rightarrow Z_{\bar{\eta}} \times \mathbb{P}_{\bar{\eta}}^{1}
$$

with $\operatorname{deg}(\varphi) \leqslant d$, then so does $\mathscr{X}_{\bar{\kappa}}^{\prime}$.

Proof. First we prove (1). We may assume $Z_{\eta}$ is the generic fiber of a reduced and irreducible projective scheme $Z_{T}$ over $T$. As $\mathscr{X}_{T}$ is normal and the schemes are projective over $T$, the rational map $\varphi$ extends to a map $\varphi_{T}$ which is defined on all codimension 1 points. By an argument of Abhyankar and Zariski [11, Lemma 2.22] for any codimension 1 point $\delta \in \mathscr{X}$, there is a birational morphism $\mu: R_{T} \rightarrow Z_{T} \times \mathbb{P}^{1}$ so that the induced rational map

$$
\varphi_{T}^{\prime}: \mathscr{X}_{T} \rightarrow R_{T}
$$

satisfies $\varphi_{T}^{\prime}(\delta)$ is a codimension 1 point and $R_{T}$ is regular at $\varphi_{T}^{\prime}(\delta)$. When $\delta$ is the generic point of $\mathscr{X}_{\kappa}^{\prime}$, then the closure of the image $R_{\kappa}^{\prime}:=\overline{\varphi_{T}^{\prime}(\delta)} \in R_{\kappa}$ satisfies the hypotheses of Matsusaka's Theorem (see [10, Theorem IV.1.6]). Therefore $R_{\kappa}^{\prime}$ is ruled, and we have produced a dominant generically finite rational map $\varphi_{\kappa}^{\prime}: \mathscr{X}_{\kappa}^{\prime} \rightarrow R_{\kappa}^{\prime}$ with $\operatorname{deg}\left(\varphi_{\kappa}^{\prime}\right) \leqslant d$.

Next, we prove (1) implies (2). There is a finite field extension $\eta \subset L \subset \bar{\eta}$ such that $\varphi$ is defined over $L$, that is, there is a map $\mathscr{X}_{L} \rightarrow Z_{L} \times \mathbb{P}^{1}$ whose base change to $\bar{\eta}$ is $\varphi$. Let $B \subset L$ be the integral closure of $A$. Let $\mathfrak{p} \subset B$ denote the ideal of a closed point over $(t=0)$ with residue field $\kappa_{B}=B / \mathfrak{p}$. This gives a map of DVRs $A \rightarrow B_{\mathfrak{p}}$. Let $S=\operatorname{Spec}\left(B_{\mathfrak{p}}\right)$ and let $\mathscr{X}_{S}:=\mathscr{X}_{T} \times_{T} S$ be the base change of $\mathscr{X}_{T}$ to $S$. (1) implies that every component of the special fiber of the normalization $\left(\mathscr{X}_{S}^{\text {norm }}\right)_{\kappa_{B}}$ admits a map to a ruled variety with degree bounded by $d$.

It remains to show that for any component $\mathscr{X}_{\kappa}^{\prime} \subset \mathscr{X}_{\kappa}$ which is geometrically reduced and geometrically irreducible (as in the statement of the theorem), 
the normal locus of $\mathscr{X}_{S}$ contains the generic point of the divisor

$$
\mathscr{X}_{\kappa_{B}}^{\prime}:=\mathscr{X}_{\kappa}^{\prime} \times_{\kappa} \kappa_{B} \subset \mathscr{X}_{S} .
$$

The assumption that $\mathscr{X}_{\kappa}^{\prime}$ is geometrically reduced implies that $\mathscr{X}_{S}$ is regular at the generic point of $\mathscr{X}_{\kappa_{B}}^{\prime}$. So in particular, $\mathscr{X}_{S}$ is normal at the generic point of $\mathscr{X}_{\kappa_{B}}^{\prime}$.

Now we prove two results about the behavior of the minimal degree map to a ruled variety. We thank the referee for providing a cleaner proof of the following lemma.

LEMMA 1.2. Let $L \supset k$ be a field extension of an algebraically closed field $k$. If $X$ is a variety over $k$ of dimension $n$, then $X$ admits a dominant generically finite rational map of degree d to a ruled variety over $k \Longleftrightarrow X_{L}:=X \times_{k} L$ admits a dominant generically finite rational map of degree $d$ to a ruled variety over $L$.

Proof. The forward direction is clear. For the converse, assume that $X_{L}$ admits a dominant generically finite rational map of degree $d$ to a ruled variety over $L$ :

$$
X_{L} \rightarrow Z_{L} \times \mathbb{P}_{L}^{1}
$$

As all of this must be defined over a finitely generated extension of $k$, we may assume that $L / k$ is finitely generated. There is an affine variety $B$ with $k(B) \cong L$. The above rational map of $L$-varieties spreads out to a dominant generically finite rational map

$$
X \times B \rightarrow Z \times \mathbb{P}_{k}^{1},
$$

where $Z$ is a variety over $B$. Since $k$ is algebraically closed, $B$ has a $k$-point $b \in B(k)$ such that $X \times\{b\}$ meets the locus where the above rational map is defined. This gives the desired dominant generically finite rational map

$$
X \times\{b\} \rightarrow Z_{b} \times \mathbb{P}_{k}^{1} .
$$

We thank François Greer and Burt Totaro for suggesting the following lemma.

LEMmA 1.3. Working over $\mathbb{C}$, let $\mathscr{X} \rightarrow T$ be a smooth projective family of varieties over a variety $T$ with geometric generic point $\bar{\eta}$. If a very general fiber $\mathscr{X}_{t}$ admits a generically finite dominant rational map of degree $d$ to a ruled variety then so does $\mathscr{X}_{\bar{\eta}}$, and every fiber admits a generically finite dominant rational map of degree $\leqslant d$ to a ruled variety. 
Proof. By [17, Lemma 2.1], there is a field isomorphism of $\varphi: \bar{\eta} \cong \mathbb{C}$ such that the fiber product $\mathscr{X}_{t} \times_{\mathbb{C}} \bar{\eta}$ is isomorphic to $\mathscr{X}_{\bar{\eta}}$ as varieties over $\bar{\eta}$. Let $\mathscr{X}_{t} \rightarrow Z \times_{\mathbb{C}}$ $\mathbb{P}_{\mathbb{C}}^{1}$ be a dominant generically finite rational map of degree $d$ to a ruled complex variety. This induces a dominant generically finite rational map of degree $d$ :

$$
\mathscr{X}_{\bar{\eta}} \cong \mathscr{X}_{t} \times_{\mathbb{C}} \bar{\eta} \rightarrow\left(Z \times_{\mathbb{C}} \mathbb{P}_{\mathbb{C}}^{1}\right) \times_{\mathbb{C}} \bar{\eta}
$$

Finally, $\left(Z \times \mathbb{P}^{1}\right) \times_{\mathbb{C}} \bar{\eta} \cong\left(Z \times_{\mathbb{C}} \bar{\eta}\right) \times \mathbb{P}^{1}$ is a ruled variety over $\bar{\eta}$, which completes the proof.

Using Theorem 1.1, we can now prove Proposition C and Corollary D.

Proof of Proposition C. Let

$$
\pi: \mathscr{X} \rightarrow T
$$

be a family of smooth projective varieties as in Proposition C. By Theorem 1.1 and Lemma 1.3 , it follows that the special fiber $\mathscr{X}_{0}$ admits a dominant and generically finite rational map

$$
\varphi: \mathscr{X}_{0} \rightarrow Y \simeq_{\text {bir }} Z \times \mathbb{P}^{1}
$$

such that $\operatorname{deg}(\varphi) \leqslant d$, and $Z$ is smooth. Consider the MRC fibration of $Z$, given by

$$
\psi: Z \rightarrow B,
$$

where $B$ is smooth and projective. Then $B$ has dimension 0,1 , or 2 . We treat each dimension separately. In the case $B$ has dimension 0 , then $Z$ is in fact rational, and we have $\operatorname{irr}\left(\mathscr{X}_{0}\right) \leqslant d$.

Now we rule out the cases where $\operatorname{dim}(B)=1$ or 2 . If $\operatorname{dim}(B)=1$, then $B$ must have positive geometric genus. But there are no dominant rational maps from $\mathscr{X}_{0}$ to such a curve $\left(\right.$ as $\left.h^{1,0}\left(\mathscr{X}_{0}\right)=0\right)$. If $\operatorname{dim}(B)=2$, then we are in the case (2) of Proposition C, so $\mathscr{X}_{0}$ is a strict Calabi-Yau threefold. Iitaka's $C_{n, m}$ conjecture, which is known for $n=\operatorname{dim}(X) \leqslant 6$ (see [3]), states

$$
0=\kappa\left(X_{0}\right) \geqslant \kappa(B)+\kappa(F),
$$

where $F$ is a general fiber of the map $X_{0} \rightarrow B$, and $\kappa$ denotes the Kodaira dimension. Neither $\kappa(F)$ nor $\kappa(B)$ are $-\infty$ as $X_{0}$ and $B$ are not uniruled. Thus $\kappa(B)=\kappa(F)=0$, and $B$ is a Kodaira dimension 0 surface. By the classification of surfaces there is a finite étale cover $B^{\prime} \rightarrow B$ such that $\omega_{B^{\prime}} \cong \mathcal{O}_{B^{\prime}}$. As $\mathscr{X}_{0}$ is simply connected, the map from $\mathscr{X}_{0}$ to $B$ factors through $B^{\prime}$. But this contradicts the fact that strict Calabi-Yau threefolds have $h^{p, 0}=0$ for $p=1,2$.

Proof of Corollary D. The quotient map from $A$ to the Kummer surface $A / \pm 1$ has degree 2 , so it suffices to prove $\operatorname{irr}(A / \pm 1)=2$. The main result of [4] can be 
rephrased as saying that a very general Kummer surface has degree of irrationality equal to 2 . It follows that one can put $A / \pm 1$ in a family over a curve such that the very general member has degree of irrationality equal to 2 . Taking a simultaneous resolution gives a family of $\mathrm{K} 3$ surfaces, so by Proposition $\mathrm{C}$ every member of the family has degree of irrationality equal to 2 .

\section{Irrationality of Fano hypersurfaces}

The goal of this section is to prove Theorem A. We follow an idea of Kollár's [9], which he used to prove nonruledness of certain Fano hypersurfaces. Kollár reduces to positive characteristic and observes that if a smooth projective variety $X$ is ruled, then no sheaf of $i$-forms $\wedge^{i} \Omega_{X}$ can contain a big line bundle. The main observation in this section (Lemma 2.3) is that if $X$ admits a separable rational map of degree $d$ to a ruled variety, then no sheaf of $i$-forms $\wedge^{i} \Omega_{X}$ can contain a line bundle which separates $2 d$ points on an open set. This allows us to directly apply Kollár's degeneration argument (albeit in a different degree range) to deduce Theorem A.

DEFINITION 2.1. Let $X$ be a variety over an algebraically closed field $k$ and let $\mathcal{M}$ be a line bundle on $X$. We say that $H^{0}(X, \mathcal{M})$ separates $\ell$ points on an open set if there is a Zariski open set $U \subset X$ such that for any distinct $\ell$ points $x_{1}, \ldots$, $x_{\ell} \in U$, there is a section $s \in H^{0}(X, \mathcal{M})$ which vanishes on $x_{1}, \ldots, x_{\ell-1}$ but not on $x_{\ell}$.

EXAMPLE 2.2. Let $X$ and $Y$ be projective varieties of dimension $n$ over an algebraically closed field $k$ and suppose there is a map

$$
\psi: X \rightarrow Y
$$

which is a dominant, generically finite, purely inseparable morphism. Then over the open set $U \subset Y$ where $\psi$ is finite, there is a bijection on $k$-points:

$$
\psi^{-1}(U)(k) \cong U(k) .
$$

As a consequence, if there is a line bundle $\mathcal{M}$ on $Y$ such that $H^{0}(Y, \mathcal{M})$ separates $\ell$ points on an open set, then $H^{0}\left(X, \psi^{*} \mathcal{M}\right)$ separates $\ell$ points on an open set in $X$.

LEMMA 2.3. Let $X$ be a projective variety over an algebraically closed field and $\mathcal{M}$ a line bundle on $X$ such that $H^{0}(X, \mathcal{M})$ separates $2 \delta$ points on an open set. Suppose that there is an injection

$$
\mathcal{M} \hookrightarrow \wedge^{i} \Omega_{X}
$$


for some $i>0$. If there is a dominant, separable, generically finite rational map

$$
\varphi: X \rightarrow Z \times \mathbb{P}^{1}
$$

to a ruled variety, then $\operatorname{deg}(\varphi) \geqslant \delta+1$.

Proof. Let $b:=\operatorname{deg}(\varphi)$. Without loss of generality, we may assume that $X$ and $Z$ are normal and $Z$ is projective. Let $\Gamma$ be the normalization of the closure of the graph of $\varphi$. This gives two regular maps $\mu, \psi$, which make the diagram commute:

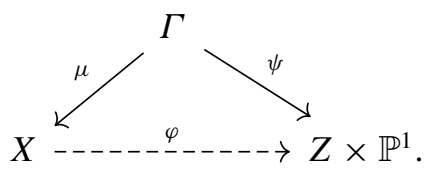

Note that $\mu$ is birational, so $\mu^{*} \mathcal{M}$ is a line bundle that separates $2 \delta$ points on an open set in $\Gamma$ and injects into $\wedge^{i} \Omega_{\Gamma}$.

Recall that there is a trace map

$$
\operatorname{Tr}_{\psi}^{i}: H^{0}\left(\Gamma, \wedge^{i} \Omega_{\Gamma}\right) \rightarrow H^{0}\left(Z \times \mathbb{P}^{1},\left(\wedge^{i} \Omega_{Z \times \mathbb{P}^{1}}\right)^{\vee \vee}\right)
$$

(see [7, Proposition 3.3] and [8]) which extends the usual trace map for finite morphisms in characteristic 0 . Over the dense open set in $Z \times \mathbb{P}^{1}$ where $\psi$ is étale, $\operatorname{Tr}_{\psi}^{i}$ corresponds to the sum over fibers. Let $\pi_{1}$ (respectively, $\pi_{2}$ ) denote the projection of $Z \times \mathbb{P}^{1}$ onto $Z$ (respectively, $\mathbb{P}^{1}$ ). By a computation,

$$
\left(\wedge^{i} \Omega_{Z \times \mathbb{P}^{1}}\right)^{\vee \vee} \cong\left(\wedge^{i}\left(\pi_{1}^{*} \Omega_{Z}\right)\right)^{\vee \vee} \oplus\left(\left(\wedge^{i-1}\left(\pi_{1}^{*} \Omega_{Z}\right)\right)^{\vee \vee} \otimes \pi_{2}^{*}\left(\Omega_{\mathbb{P}^{1}}\right)\right) .
$$

So any section of $H^{0}\left(Z \times \mathbb{P}^{1},\left(\wedge^{i} \Omega_{Z \times \mathbb{P}^{1}}\right)^{\vee \vee}\right)$ is necessarily constant along $\{z\} \times \mathbb{P}^{1}$ for every $z \in Z$.

However, if we take a general point $z \in Z$ and two general points $z_{1}, z_{2} \in z \times \mathbb{P}^{1}$, then the preimage of these points will consist of $2 b$ distinct points

$$
\psi^{-1}\left(\left\{z_{1}, z_{2}\right\}\right)=\left\{\gamma_{1}, \ldots, \gamma_{2 b}\right\}
$$

If $b \leqslant \delta$, then there will be a section

$$
\alpha \in H^{0}\left(\Gamma, \mu^{*}(\mathcal{M})\right)
$$

which vanishes on $\gamma_{1}, \ldots, \gamma_{2 b-1}$ but does not vanish on $\gamma_{2 b}$. Tracing $\alpha$ as an $i$-form gives a section

$$
\operatorname{Tr}_{\psi}^{i}(\alpha) \in H^{0}\left(Z \times \mathbb{P}^{1},\left(\wedge^{i} \Omega_{Z \times \mathbb{P}^{1}}\right)^{\vee \vee}\right),
$$

which vanishes on $z_{1}$ but not on $z_{2}$, yielding a contradiction. Therefore $b \geqslant \delta+1$. 
CONSTRUCTION 2.4. Now we recall Kollár's degeneration argument [9, Section 5] (the construction of the family used in the argument is due to Mori [13]). Let $R$ be a DVR with an algebraically closed residue field $\kappa$ of characteristic $p$ and fraction field $\eta$ which is countable of characteristic 0 . Kollár shows that over $T=\operatorname{Spec}(R)$, one can construct an irreducible normal variety

$$
\pi: \mathscr{X} \rightarrow T
$$

such that $\mathscr{X}_{\eta} \subset \mathbb{P}_{\eta}^{n+1}$ is a hypersurface of degree $p e$ and $\mathscr{X}_{\kappa}$ is a reduced degree $p$ inseparable cover of a smooth degree $e$ hypersurface $Y \subset \mathbb{P}_{\kappa}^{n+1}$ with 'simple' singularities. Kollár gives an explicit resolution of singularities of $\mathscr{X}_{\kappa}$ :

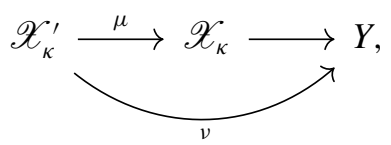

Assuming $n \geqslant 3$, Kollár shows that $\mathcal{M}:=v^{*}\left(\mathcal{O}_{\mathbb{P}_{k}^{n+1}}(p e+e-n-2)\right)$ injects into $\wedge^{n-1} \Omega_{\mathscr{X}_{\kappa}^{\prime}}$.

Having recalled Kollár's construction, we are ready to prove the following lemma:

Proposition 2.5. Let $X \subset \mathbb{P}_{\mathbb{C}}^{n+1}$ be a very general hypersurface of degree pe. If

$$
\varphi: X \rightarrow Z \times \mathbb{P}_{\mathbb{C}}^{1}
$$

is a dominant generically finite rational map, then

$$
\operatorname{deg}(\varphi) \geqslant \min \{\lfloor(p e+e-n) / 2\rfloor, p\} .
$$

Proof. Consider a hypersurface $\mathscr{X}_{\eta} \subset \mathbb{P}_{\mathbb{C}}^{n+1}$ of degree pe degenerating to a $p$-fold cover of a degree $e$ hypersurface, as in Kollár's construction above. We know that the map $v$ in $(\star)$ is purely inseparable, and the sections of

$$
H^{0}\left(\mathbb{P}_{\kappa}^{n+1}, \mathcal{O}_{\mathbb{P}^{n+1}}(p e+e-n-2)\right)
$$

separate every set of $p e+e-n-2$ points. So by Example 2.2 we see that $H^{0}\left(\mathscr{X}_{\kappa}^{\prime}, \mathcal{M}\right)$ separates $p e+e-n-2$ general points. Lemma 2.3 then implies that there are no dominant separable generically finite rational maps from $\mathscr{X}_{\kappa}$ to a ruled variety of degree $<\lfloor(p e+e-n) / 2\rfloor$.

Since $p$ must divide the degree of any inseparable map, any dominant generically finite rational map from $\mathscr{X}_{\kappa}$ to a ruled variety must have degree $\geqslant \min \{\lfloor(p e+e-n) / 2\rfloor, p\}$. Thus, Theorem 1.1 implies that any dominant 
generically finite rational map from $\mathscr{X}_{\bar{\eta}}$ to a ruled variety must have degree $\geqslant \min \{\lfloor(p e+e-n) / 2\rfloor, p\}$. By base changing $\bar{\eta}$ to $\mathbb{C}$ and applying Lemma 1.2 it follows that every dominant rational map from $\mathscr{X}_{\mathbb{C}}$ to a ruled variety has the same lower bound. By Lemma 1.3, the same holds true for a very general hypersurface.

Now we are ready to prove Theorem A.

Proof of Theorem A. The idea is to degenerate $X$ to the union of a very general hypersurface of degree $p e$ and $d-p e$ hyperplanes and then apply Lemma 2.5 and Theorem 1.1.

Theorem A is trivial for $n<14$, so assume $n \geqslant 14$. Let $d$ and $n$ satisfy

$$
d \geqslant n+1-\sqrt{n+2} / 4
$$

as in the statement of the theorem. Bertrand's postulate says that given any $m>1$, there is always a prime $p$ such that $m<p<2 m$. This implies there exists a prime $p$ with

$$
\frac{\sqrt{n+2}}{4} \leqslant p \leqslant \frac{\sqrt{n+2}}{2}
$$

Fix such a prime $p$ and let $f$ be the remainder of $d$ modulo $p$, so that

$$
d=p e+f .
$$

First we have

$$
\begin{aligned}
p e-n & =d-f-n \\
& \geqslant 2-\frac{\sqrt{n+2}}{4}-p \quad(\text { by }(1) \text { using that } f \leqslant p-1) \\
& \geqslant 2-2 p \quad(\text { by }(2)) .
\end{aligned}
$$

This yields the following inequality:

$$
\begin{aligned}
p(p e+e-n) & =n+(p+1)(p e-n) \\
& \geqslant n+(p+1)(2-2 p) \quad(\text { by }(3)) \\
& =n-2 p^{2}+2 \\
& \geqslant 2 p^{2} \quad(\text { by }(2)) .
\end{aligned}
$$

Thus,

$$
p e+e-n \geqslant 2 p .
$$

Let $W$ be a very general hypersurface of degree pe. By Proposition 2.5 and inequality (5), any dominant, generically finite, rational map from $W$ to a ruled 
variety has degree at least $p$. Consider a pencil of degree $d$ hypersurfaces, which contains both a smooth hypersurface and the union of $W$ and $f$ hyperplanes. By Theorem 1.1 and Lemma 1.3, the very general member of this pencil admits no dominant generically finite rational maps to a ruled variety with degree less than $p$. Applying Lemma 1.3 to the universal family of smooth degree $d$ hypersurfaces, if $X$ is a very general degree $d$ hypersurface, then every dominant generically finite rational map from $X$ to a ruled variety has degree at least $p$. The theorem follows from inequality (2).

REMARK 2.6. We note that the above bound is not optimal. One might hope that the bound on $\operatorname{irr}(X)$ can be improved to a linear bound. If one applies Proposition 2.5, the first new bounds occur using $p=5, n=34$ and $d=35$. In other words, a very general degree 35 hypersurface $X \subset \mathbb{P}_{\mathbb{C}}^{35}$ has $\operatorname{irr}(X) \geqslant 4$.

\section{Acknowledgements}

We are grateful for valuable conversations and correspondences with Iacopo Brivio, Lawrence Ein, François Greer, Kiran Kedlaya, János Kollár, Robert Lazarsfeld, James McKernan, John Ottem, John Sheridan, Jason Starr, Burt Totaro and Ruijie Yang. We would also like to thank the referee for a detailed reading and many helpful suggestions.

The first author's research is partially supported by the National Science Foundation under the Stony Brook/Simons Center for Geometry and Physics RTG grant DMS-1547145.

\section{Conflict of Interest: None.}

\section{References}

[1] F. Bastianelli, P. De Poi, L. Ein, R. Lazarsfeld and B. Ullery, 'Measures of irrationality for hypersurfaces of large degree', Compos. Math. 153 (2017), 2368-2393.

[2] R. Beheshti and E. Riedl, 'Linear subspaces of hypersurfaces', Preprint, 2019, arXiv:1903.0 2481.

[3] C. Birkar, 'The Iitaka conjecture $C_{n, m}$ in dimension six', Compos. Math. 145 (2009), 1442-1446.

[4] N. Chen, 'Degree of irrationality of very general abelian surfaces', Algebra Number Theory 13 (2019), 2191-2198.

[5] B. Hassett, A. Pirutka and Y. Tschinkel, 'Stable rationality of quadric surface bundles over surfaces', Acta Math. 220 (2018), 341-365.

[6] V. A. Iskovskih and J. I. Manin, 'Three-dimensional quartics and counterexamples to the Lüroth problem', Mat. Sb. 86(128) (1971), 140-166. 
[7] A. J. de Jong and J. Starr, 'Cubic fourfolds and spaces of rational curves', Illinois J. Math. 48 (2004), 415-450.

[8] A. J. de Jong and J. Starr, 'Erratum: cubic fourfolds and spaces of rational curves', Illinois J. Math. 52 (2008), 345-346.

[9] J. Kollár, 'Nonrational hypersurfaces', J. Amer. Math. Soc. 8 (1995), 241-249.

[10] J. Kollár, Rational Curves on Algebraic Varieties, Ergebnisse der Mathematik und ihrer Grenzgebiete, 32 (Springer, Berlin, 1996).

[11] J. Kollár, Singularities of the Minimal Model Program, vol. 200. (Cambridge University Press, Cambridge, 2013).

[12] M. Kontsevich and Y. Tschinkel, 'Specialization of birational types', Invent. Math. 217 (2019), 415-432.

[13] S. Mori, 'On a generalization of complete intersections', J. Math. Kyoto Univ. 15 (1975), 619-646.

[14] J. Nicaise and E. Shinder, 'The motivic nearby fiber and degeneration of stable rationality', Invent. Math. 217 (2019), 377-413.

[15] S. Schreieder, 'Stably irrational hypersurfaces of small slopes', J. Amer. Math. Soc. 32 (2019), 1171-1199.

[16] B. Totaro, 'Hypersurfaces that are not stably rational', J. Amer. Math. Soc. 29 (2016), 883-891.

[17] C. Vial, 'Algebraic cycles and fibrations', Doc. Math. 18 (2013), 1521-1553. 